\title{
Sound Intensity Distribution Around Organ Pipe
}

\author{
Piotr ODYA, Józef KOTUS, Maciej SZCZODRAK, Bożena KOSTEK \\ Faculty of Electronics, Telecommunications, and Informatics \\ Gdańsk University of Technology \\ Narutowicza 11/12, 80-233 Gdańsk, Poland; \\ e-mail: \{piotrod, joseph,szczodry\}@sound.eti.pg.gda.pl,bokostek@audioacoustics.org
}

(received March 22, 2016; accepted July 30, 2016)

\begin{abstract}
The aim of the paper was to compare acoustic field around the open and stopped organ pipes. The wooden organ pipe was located in the anechoic chamber and activated with a constant air flow, produced by an external air-compressor. Thus, a long-term steady state response was possible to obtain. Multichannel acoustic vector sensor was used to measure the sound intensity distribution of radiated acoustic energy. Measurements have been carried out on a defined fixed grid of points. A specialized Cartesian robot allowed for a precise positioning of the acoustic probe. The resulted data were processed in order to obtain and visualize the sound intensity distribution around the pipe, taking into account the type of the organ pipe, frequency of the generated sound, the sound pressure level and the direction of acoustic energy propagation. For the open pipe, an additional sound source was identified at the top of the pipe. In this case, the streamlines in front of the pipe are propagated horizontally and in a greater distance than in a case of the stopped pipe, moreover they are directed downwards. For the stopped pipe, the streamlines of the acoustic flow were directed upwards. The results for both pipe types were compared and discussed in the paper.
\end{abstract}

Keywords: organ pipe; acoustic vector sensor; sound intensity; Cartesian robot.

\section{Introduction}

The main aim of this research study is to prepare a measurement setup and analyze the resulted acoustic field around the open and stopped organ pipes using the sound intensity technique. A similar methodology was applied by the authors for the measurements and visualization of sound intensity around a human head simulator in a free field (KotUs, Kostek, 2015). The authors applied 3D Acoustic Vector Sensor for the sound intensity measurements, which was shown in detail in another recent publications (SzCZODRAK et al., 2016; Kotus et al., 2016). The methodology used for the purpose of this paper along with the equipment applied in the research were described in detail in Secs. 2 and 3 as they needed a very careful designing.

Issues related to the acoustic behavior of organ pipes has been investigated by researchers since many decades (Coltman, 1968; Fletcher, Thwaites, 1983; Steenbrugge, 2011, VAn De Perre, 2011). One of the main researched problems was to understand how the sound is generated. Numerical methods of modeling organ pipes were verified using different forms of measurement techniques, e.g. microphones, laser Doppler anemometry, laser Doppler vibrometry (VAN DE PERRE, 2011) or Particle Image Anemometry (MiCKIEWICZ, 2014). Another paper by MiCKIEWICZ (2015) presents experimental results of the visualization of the nonlinear aeroacoustic sound generation phenomena occurring in organ flue pipe. The phaselocked particle image velocimetry technique is applied to visualize the mixed velocity field in the transparent organ flue pipe model made from Plexiglas.

However, the field of observation was often limited to the pipe mouth. Consequently, flow mechanisms in that region, as well as flow fields evolution, are well researched, but the complexity of the sound generation phenomena and especially the acoustic radiation of the pipes seems still to be the open and active research topic (Rucz, 2015). Coltman (1969) describes his observation of the radiation pattern of the double-ended organ pipe, but results are limited only to the sound pressure in a particular radius. Similarly, FLETCHER (1976) calculates and measures sound 
pressure levels for different types of pipes and different frequency modes. Directivity patterns obtained using a microphone array can be found in the study of KUANG et al. (2015). The authors of this paper concentrated on the Sheng pipes, but diagrams for the labial organ pipes are also presented. Lately, another point of interest arose, namely building scientific background behind the design in organ building practice (RUCZ, 2015; Rucz et al., 2013, 2014, 2015). As pointed out by Rucz (2015) in his Ph.D. thesis booklet, the sound generation mechanism of organ pipes is a complex process involving acoustical, mechanical and fluid dynamical phenomena inherently and non-linearly coupled.

In conventional acoustic metrology, the analysis of acoustic fields concerns the distribution of pressure levels (scalar variable), however in a real acoustic field, both the scalar (acoustic pressure) and vector (acoustic particle velocity) effects are closely related (WEYNA, 2014). Only when the acoustic field is described by both the potential and kinetic energies, it is possible to understand the mechanisms of propagation, diffraction and scattering of acoustic waves on obstacles, as a form of energy image (WEYNA, 2003). The attempt to visualize the acoustic field around the organ pipe was a goal of the research process presented by the authors. The sound intensity measurement technique was utilized to investigate how the energy propagates around the organ pipes. It is one of the most interesting techniques employed in acoustic metrology as well as for solving vibroacoustic problems, as it simplifies measurement techniques, thus effectively replacing conventional methods.

Sound intensity is a measure of the flow of acoustic energy in a sound field (FAHY, 1995; DE BREE, 2003; Gauthier et al., 2015; JACOBSEn, 2011; JACobsen, De Bree, 2005; NAgAta et al., 2005; PolyCHRONOPOUlos et al., 2014). More precisely, sound intensity $I$ is a vector quantity defined as the time average of the net flow of sound energy through unit area in the direction perpendicular to the area. Fahy's monograph "Sound Intensity" brings a thorough description of the history of the development of sound intensity measurement (FAHY, 1995).

Acoustic particle velocity may be measured using sound intensity probe, which can be then employed for collecting data to visualize all the phenomena occurring in the investigated acoustic vector fields, in both the near field and 3-dimensional space. The visualization of acoustic energy flow in real-life acoustic 3D space fields can explain many of energetic effects (scattering, vortex flow, shielding area, etc.) (WEYNA, 2010; WEyna, MickieWiCZ, 2014).

\section{Sound intensity determination}

One of the important elements of the measurement setup is the sound intensity probe. In all measurement sessions, the Acoustic Vector Sensor (AVS) was employed. This is a $p-u$ (pressure $(p)$ and particle velocity $(u)$ ) type of sound intensity probe which makes possible to simultaneously measure acoustic pressure (scalar) and particle velocity (vector component) in three orthogonally oriented directions.

To recall the basic notions, sound intensity is the average rate at which sound energy is transmitted through a unit area perpendicular to the specified direction at the considered point. Intensity in a certain direction is the product of sound pressure (scalar) $p(t)$ and the particle velocity (vector) component in that direction $u(t)$. The time-averaged intensity $I$ in a single direction is given by Eq. (1):

$$
I=\frac{1}{T} \int_{T} p(t) u(t) \mathrm{d} t .
$$

Using the presented AVS probe, the particular sound intensity components can be obtained with ease based on Eq. (1). The sound intensity vector in three dimensions is composed of the acoustic intensities in three orthogonal directions $(x, y, z)$ according to Eq. (2):

$$
\mathbf{I}=I_{x} \mathbf{e}_{x}+I_{y} \mathbf{e}_{y}+I_{z} \mathbf{e}_{z} .
$$

In the AVS probe, the particle velocity components are determined using two miniature resistive strips of platinum that are heated up to approximately $200^{\circ} \mathrm{C}$ to provide temperature difference. The sensor itself is miniscule: typical dimensions of the heated wire are $5 \mu \mathrm{m}$ in diameter and 1 to $3 \mathrm{~mm}$ in length, thus giving a nearly pin-point measurement. It operates in a flow range of $10 \mathrm{~nm} / \mathrm{s}$ up to about $1 \mathrm{~m} / \mathrm{s}$. Each particle velocity sensor is sensitive in only one direction, therefore, three orthogonally placed particle velocity transducers are built into the sensor. In combination with a pressure microphone, the sound field in a single point is fully characterized, and the acoustic intensity vector, which is the product of pressure and particle velocity, can be determined. The intensity vector indicates the level and direction of the energy flow in a plane. With a compact probe, the full three dimensional sound intensity vector can be determined within the audible frequency range from $20 \mathrm{~Hz}$ up to $20 \mathrm{kHz}$ (DE Bree, 2003; Jacobsen, 2011). However, during carried out measurements we were focused on low frequency range, because the acoustic energy radiated by the organ pipes was present in this region.

Sound intensity calculation can be performed in the time domain or in the frequency domain. Due to the fact that the multi-harmonic signal was generated by the organ pipe, a method for calculating sound intensity in the frequency domain was applied (KoTUs, $2015)$. In the applied algorithm of sound intensity calculation, the time average $T$ (Eq. (1)) was 4096 samples (with the sampling frequency $44.1 \mathrm{kHz}$ ). It means that the direction of the sound source was updated 


\begin{tabular}{|c|c|c|c|c|}
\hline $\begin{array}{c}\text { Acoustic signals } \\
\text { buffering }(p, x, y, z)\end{array}$ & $\begin{array}{l}4 \times \text { FFT with } \\
\text { overlapping }\end{array}$ & $\begin{array}{c}\text { Amplitude and phase } \\
\text { correction }\end{array}$ & $\begin{array}{l}\text { Intensity components } \\
\text { calculation } \\
\text { in frequency domain }\end{array}$ & $\begin{array}{c}\text { Data visualization } \\
\text { (ParaView) }\end{array}$ \\
\hline
\end{tabular}

Fig. 1. The block diagram of the algorithm applied for sound intensity determination.

more than 10 times per second. The intensity measurement in a single point takes 1 second.

The block diagram of the algorithm applied for sound intensity determination is depicted in Fig. 1. The sound intensity calculation algorithm acquired signals delivered by the multichannel acoustic vector sensor. As mentioned before, it produced the following signals: sound pressure $p$ and three orthogonal particle velocity components $\left(u_{x}, u_{y}, u_{z}\right)$.

After triggering the calculation by the synchronization signal (see Subsec. 3.1 for details), the FFT calculation was performed. The Hanning window was applied. Subsequently, the 4096 point FFT calculation for each signal was performed with the sampling frequency equal to $44.1 \mathrm{kS} / \mathrm{s}$ (frequency resolution: $10.8 \mathrm{~Hz}$ ). Such parameters provide sufficient spectral resolution for sound intensity calculations. The overlap was equal to $50 \%$. The FFT calculation (marked in Eq. (4) as: $\Im$ ) was performed for each acoustic component $\left(p, u_{x}, u_{y}, u_{z}\right)$, separately. This operation yields transformed signals: $X_{p}(i), X_{u x}(i), X_{u y}(\mathrm{i})$, $X_{u z}(i)$ where $i$ (ranging from 0 to 4095) denotes the index of the spectral bin.

The matrix $\mathbf{X}$ (Eq. (3)) contains information about the arrival direction for every spectral component of the signal.

$$
\mathbf{X}=\left[\begin{array}{lll}
X_{p}(i) & X_{u x}(i) X_{u y}(i) X_{u z}(i)
\end{array}\right]
$$

or

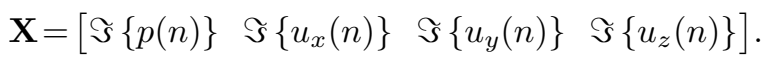

The sound intensity vector is defined and calculated according to Eq. (5).

$$
\mathbf{I}(i)=\left[\begin{array}{c}
I_{x}(i) \\
I_{y}(i) \\
I_{z}(i)
\end{array}\right]=\left[\begin{array}{c}
X_{p}(i) \cdot \overline{X_{u x}(i)} \\
X_{p}(i) \cdot \overline{X_{u y}(i)} \\
X_{p}(i) \cdot \overline{X_{u z}(i)}
\end{array}\right]
$$

where $I_{x}(i)$ - sound intensity component for $x$ direction for the $i$-th spectral component, $X_{p}(i)-$ coefficients of complex spectrum for $i$-th spectral component for acoustic pressure signal, $\overline{X_{u x}(i)}$, conjugated spectrum coefficients for particle velocity in $x$ direction.

Finally, the obtained sound intensity values were used to prepare visualization of the sound intensity distribution around organ pipes using ParaView software (AHrens et al., 2005). Other details of the algorithm applied for sound intensity determination, especially related to phase and amplitude correction, can be found in the earlier work prepared by the authors (Kotus, Kostek, 2015; Kotus, 2015). Intensity magnitude was calculated using formula (6):

$$
L_{i}=10 \log _{10}\left(I / I_{0}\right),
$$

where $I$ is defined in Eq. (2) and $I_{0}=1 \mathrm{pW} / \mathrm{m}^{2}$ is the reference sound intensity.

\section{Measurement framework}

\subsection{Cartesian robot}

The measurement framework consists in the Cartesian robot, AVS probe, audio recorder ZOOM H6 and software dedicated to data acquisition as well as their analysis. The robot can place the measurement probe with a positioning accuracy of $200 \mu \mathrm{m}$. Ranges of motion are as follows: $\mathrm{X}-1850, \mathrm{Y}-2000$ and $\mathrm{Z}-$ $1540 \mathrm{~mm}$. The movement speed for a particular axis is: $\mathrm{X}-50, \mathrm{Y}-50, \mathrm{Z}-15 \mathrm{~mm} / \mathrm{s}$.

In the movement phase, the sensor position is checked. If the sensor position is correct, the linear drive motors switch off to avoid unwanted noise and vibrations that could disturb the measurements. Otherwise, the positioning procedure is performed again. During the sound intensity measurement, the additional synchronization signal was generated in a separate channel. It was recorded simultaneously with measurement signals. The synchronization signal (sync) was used during the offline analysis. It enabled unambiguous assignment of every measurement result to the position in which it was captured. After finishing the current measurement, the linear drive motors were switched on again, and the measurement sensor was moved to the next position. The procedure of positioning and measuring was finished when the measurements were performed in all positions. In the next step, the recorded signals were used for offline calculations of sound intensity values. More details of this process are presented in Subsec. 3.4.

\subsection{Organ pipes}

A set of two organ pipes available at the Multimedia Systems Department, Gdańsk University of Technology (see Fig. 2a), was adapted to the needs of the measurement setup. It consists of the two organ pipes (metal and wooden) and a stand which receives compressed air. The stand was altered to increase the measurements range along the $z$-axis and to enable to use an air compressor (Kotus et al., 2015a; 2015b). 
a)

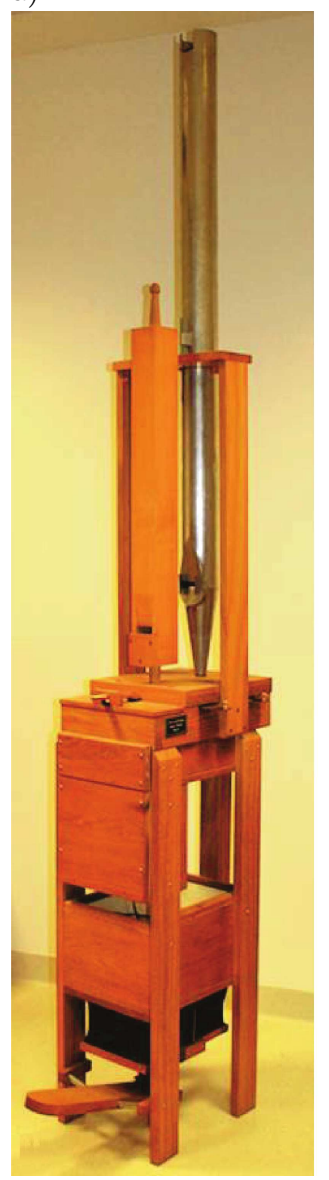

b)

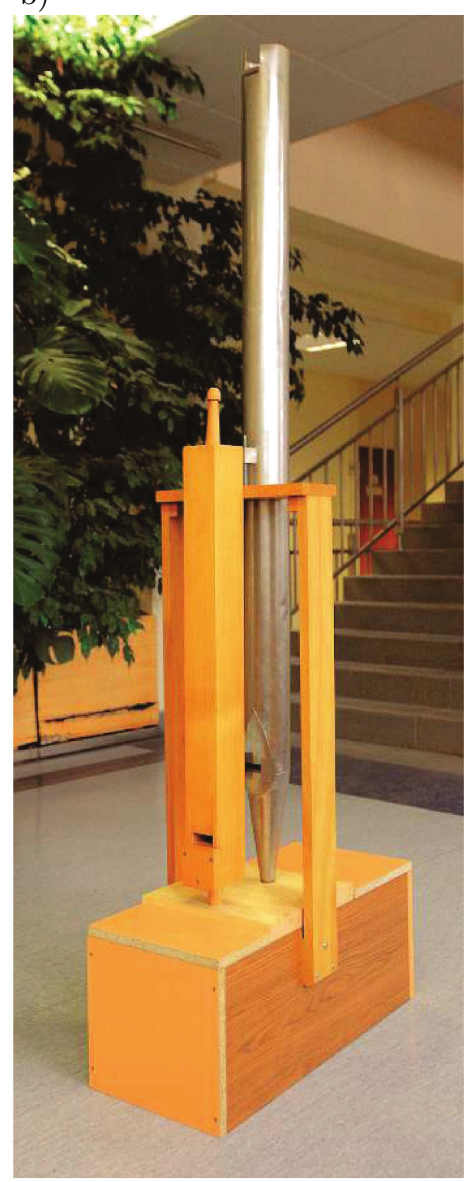

Fig. 2. Set of organ pipes with old (a) and new stand (b).

The new stand is presented in Fig. 2b). In this way, it was possible to reduce the height of the set to about $135 \mathrm{~cm}$ for the wooden pipe (previously: $215 \mathrm{~cm}$ ). The wooden organ pipe (Bourdon) is originally stopped, its full length is $88 \mathrm{~cm}$ (99 cm with a tuning stopper). This pipe has a rectangle cross-section of $5.4 \times 6.6 \mathrm{~cm}$. It was decided to use the same pipe (with and without the tuning stopper) to obtain results that can show clearly the differences in sound intensity distributions in case of the two organ pipe types.

The fundamental frequencies of the pipe can be obtained using equations: (7) for the stopped pipe and (8) for the open pipe (FLETCHeR, Rossing, 1998)

$$
\begin{aligned}
& f_{1}=\frac{1}{4} \cdot \frac{c}{L}, \\
& f_{1}=\frac{1}{2} \cdot \frac{c}{L},
\end{aligned}
$$

where $c$ is the velocity of sound in air at the room temperature at which the organ is to be played $(\sim 340 \mathrm{~m} / \mathrm{s}$ at $20^{\circ} \mathrm{C}$ ), $L$ is the effective length of the pipe (between a languid and a pipe end).

The pipe has the effective length of $70 \mathrm{~cm}$, thus the fundamental frequencies are equal to $120 \mathrm{~Hz}$ for the stopped and $240 \mathrm{~Hz}$ for the open pipe. During the measurements, it turned out that these values are lower - about 104 and $190 \mathrm{~Hz}$ (respectively). Differences can result from temperatures values below $20^{\circ} \mathrm{C}$ during the measurements. Furthermore, the effective length of the stopped pipe is shorter than $70 \mathrm{~cm}$ - the tuning stopper reduces the length by about 5 to $6 \mathrm{~cm}$.

\subsection{Measurements setup}

The measurement setup block diagram is presented in Fig. 3. The pipe was located inside the Cartesian robot, in the middle of a horizontal plane (Fig. 4). The location along the $z$-axis was chosen in such a way that the sound probe for the $z=0$ value was located near the lip but it should also be possible to measure sound distribution above the pipe upper end. Finally, the probe was located close to the lip for the following coordinates: $x=1000, y=1000$ and $z=0$. In such position, the probe was about $3 \mathrm{~cm}$ above the lips and about $9 \mathrm{~cm}$ from the pipe body (Fig. 5). In turn, the lowest sound probe location above the pipe $(z=-800)$ was about $10 \mathrm{~cm}$ from the upper end of the open pipe (Fig. 6).

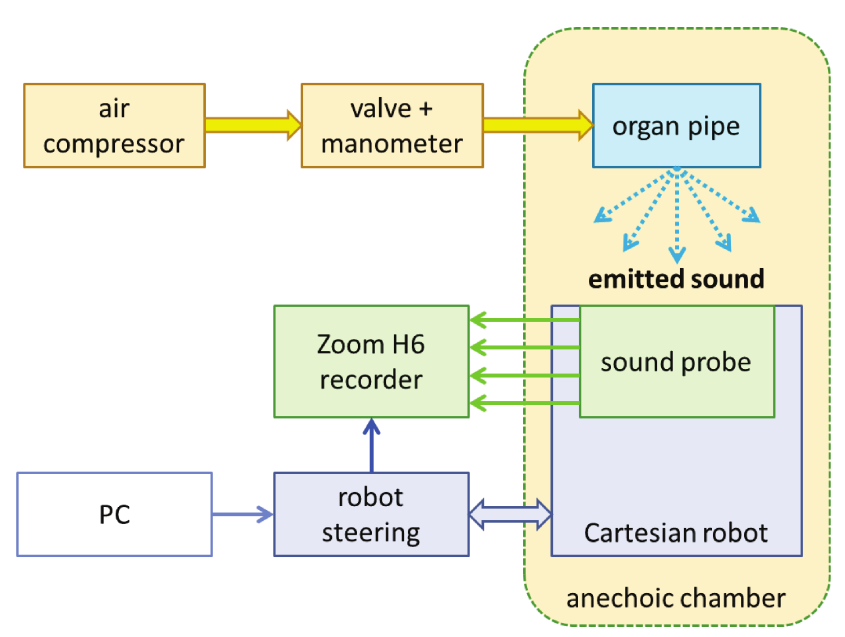

Fig. 3. Block diagram of the measurement setup.

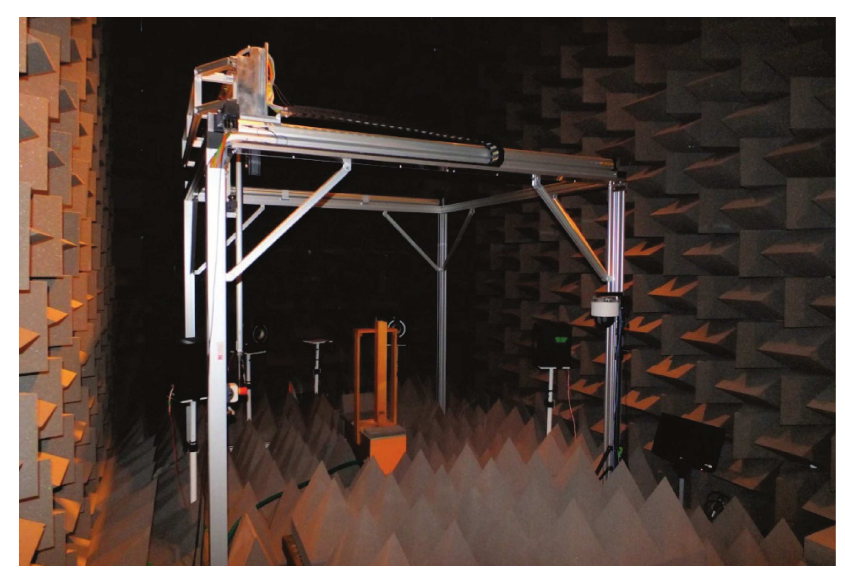

Fig. 4. Cartesian robot and the organ pipe installed in the anechoic chamber. 


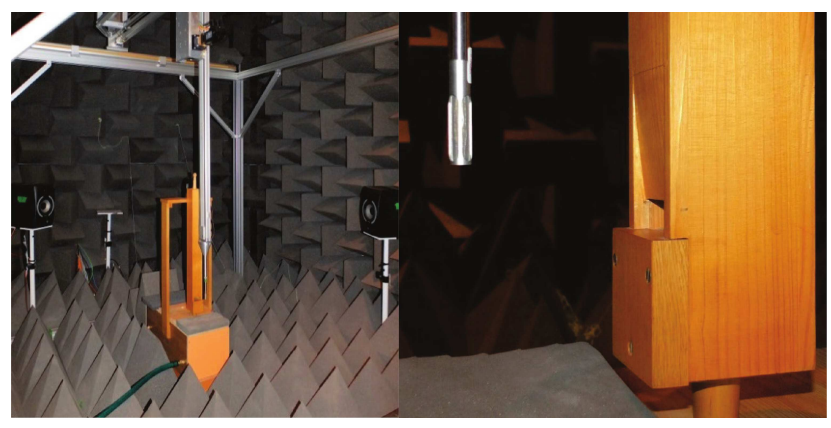

Fig. 5. Sound intensity probe in the position closest to the lips.

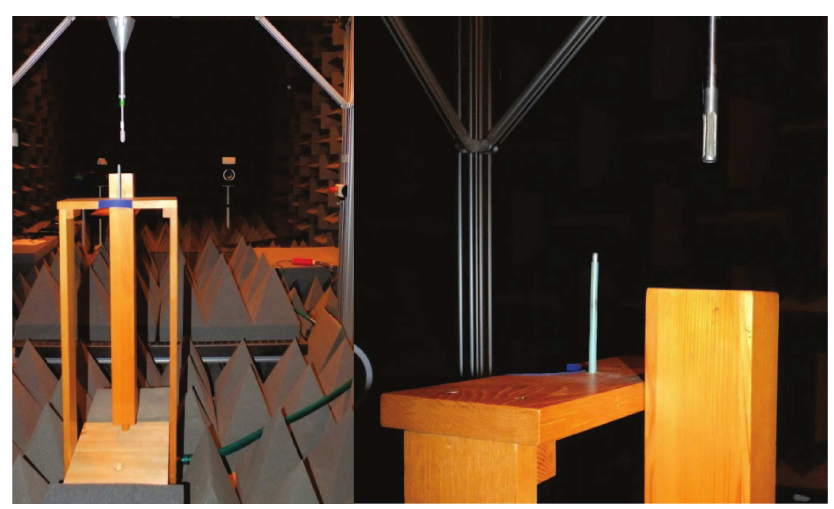

Fig. 6. Sound intensity probe in the lowest position above the upper end of the open pipe.

The pipe received air using the $15 \mathrm{~m}$ long hose. Thus, it was possible to place the air compressor in a room separated from the anechoic chamber. Such location of the compressor allowed for eliminating the impact of unwanted sounds generated by the compressor on the measurement results. The air pressure activating the organ pipe was controlled using a valve and a manometer mounted on the hose. The air pressure value was set to achieve strong and clear sound. With air pressure at a low level, the pipes played quietly and indistinctly, while too strong air pressure caused the overblowing phenomenon. The sound level meter Investigator 2260 Bruel \& Kjaer was used to control the SPL of the sound components, especially the fundamental component. The SPL measured (during the steady state response) in the position where the probe was close to the lip was equal to $99.9 \mathrm{~dB}$ for the stopped pipe and $97.8 \mathrm{~dB}$ for the open pipe. The SPL above the upper end of the open pipe was equal to $98.3 \mathrm{~dB}$. The microphone was placed in the same position as the sound probe in Fig. 6 .

As mentioned before, the acoustic vector sensor has four signal outputs - acoustic pressure and three orthogonally placed acoustic velocity components. In addition, it is also required to record the sync signal from the Cartesian robot steering. The fifth signal is used to mark the points in time when the arm of the Cartesian robot is fixed in the desired location.
The audio recorder ZOOM H6 was used during the experiments. With a connected EXH-6 Dual XLR/TRS Combo capsule it allows for recording up to six channels with sampling frequency up to $96 \mathrm{kHz}$ and 24-bit resolution on a SD card. The files are stored in the WAVE format. During the measurements, the sample rate was set to $44.1 \mathrm{kHz}$, resolution to 24 bit. The parameters of the recorder were verified by the multichannel audio analyzer APx585 before the measurements. Results proved that the recorder preserves high quality of sound, e.g. frequency characteristics ripples are below $0.4 \mathrm{~dB}$ for all channels in $50-15000 \mathrm{~Hz}$ range (Fig. 7). Similarly, THD+N values are below $0.015 \%$.

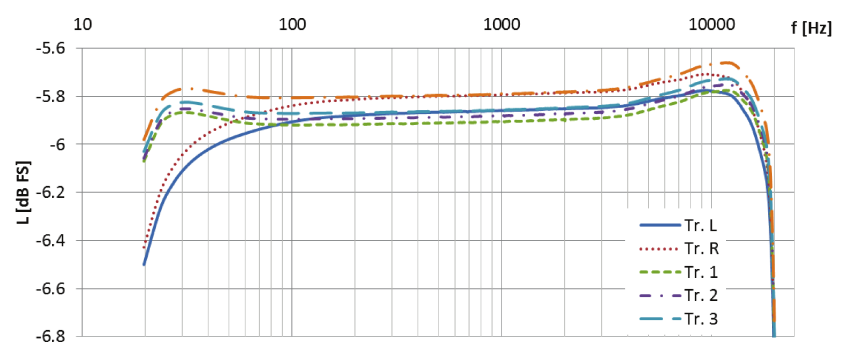

Fig. 7. Frequency characteristics of Zoom H6 inputs.

During each measurement session, the probe was moving only along the $x$ - and the $z$-axes: the $y$-axis position remained unchanged. The distance between measurement points was set to 100 , i.e. $10 \mathrm{~cm}$. The simplified movement path is shown in Fig. 8. After completing the measurement session, the position of the robot arm was changed on the $y$-axis and the next session was started. The $y$-axis values were changed between 50 and 1950, the step was also set to 100 . In addition, the measurement for $y=1000$ value was made - a plane for this value can be described as the plane of symmetry of the pipe.

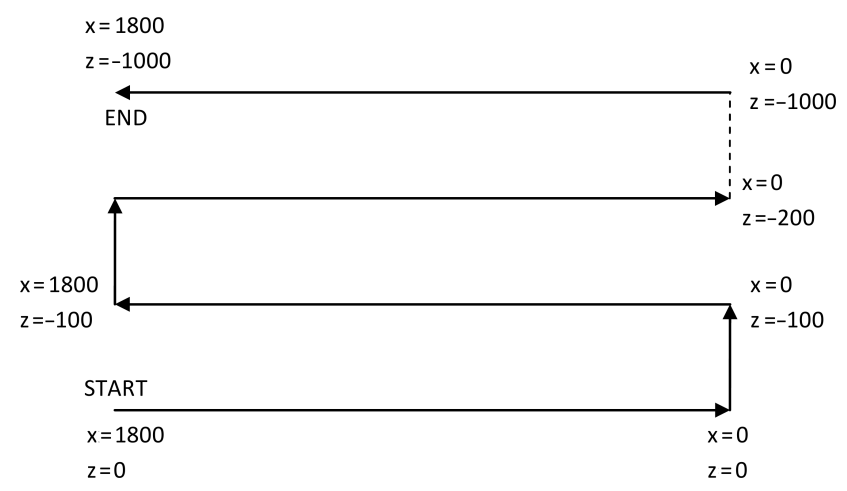

Fig. 8. A simplified movement path of the vector sensor during each measurement session.

Obviously, it was inevitable to omit the area where the pipe (with the stand) was located. A cuboid between following coordinates was excluded from the measurement: $x$ values between 700 and 900, $y$ values between 850 and 1150 and $z$ values between 0 and 
-700. The measurement sessions were divided in this area into three parts: in front of the pipe, behind it and above it.

The total number of measurement points for the open pipe was equal to 4269, whereas for the stopped pipe 4264 points were used. Five points near the tuning stopper were omitted because of a risk that the probe may interfere with the upper part of the stopper.

\subsection{Sound intensity calculation}

On the basis of the obtained recordings sound intensity vectors for all measurements points were calculated. As mentioned before, intensity vectors were calculated in the frequency domain. In Fig. 9 the energy distribution in the frequency domain for both organ pipes are shown. Spectral components marked by squares and triangles were used to calculate sound intensity values. For open pipe, the frequency of the FFT components were: $193.8 \mathrm{~Hz}, 376.8 \mathrm{~Hz}$ and $570.6 \mathrm{~Hz}$. For the stopped variation of the pipe, the frequency of FFT components were: $107.7 \mathrm{~Hz}, 312.2 \mathrm{~Hz}$ and $516.8 \mathrm{~Hz}$. Obtained values were used to prepare two kind of visualizations of sound field distribution around the organ pipe for all measurement points. The difference in energy distribution in spectral domain for both organ pipes is clearly noticeable. Those differences arise mainly from the construction of considered pipes (see Subsec. 3.2 for details). For the stopped organ pipe we observed high level for 3 and 5 harmonics. For the open organ pipe sound level for higher harmonics decreases exponentially. Next difference was observed in the sound pressure level measured in neutral position (see Fig. 5 in Subsec. 3.3 for details). Stopped organ pipe generates higher SPL than the open one. Other differences were observed in radiation directivity and in intensity distribution around considered organ pipes. For better presentation of obtained results and for convenient comparison of directivity radiation for measured instruments several types of visualizations were prepared.

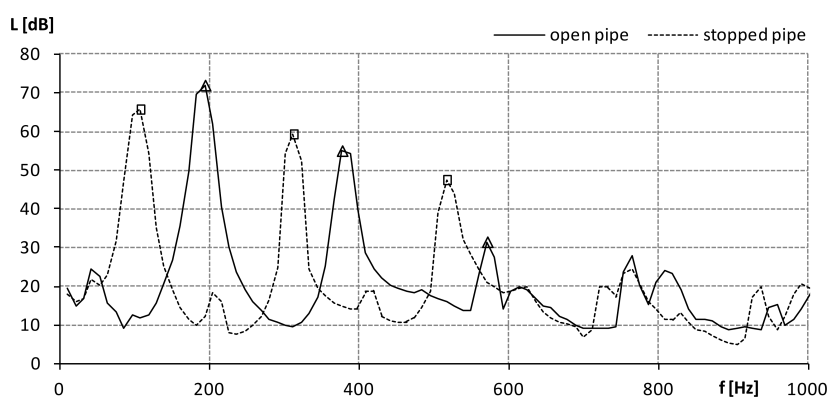

Fig. 9. Spectral energy distribution for both kind of organ pipes. Additional markers were used to indicate the frequency components used for calculating sound intensity vectors for considered instruments, squares and triangles for stopped and open pipe respectively.
The main types of visualizations are the arrows which indicate the direction of flow of acoustic energy and streamline technique to present the direction of acoustic energy flow.

\section{Results}

Based on the measurement results, the visualization was prepared and is presented in this section. For both types of organ pipe, open and stopped, the detailed discussion of the resultant sound intensity field is provided. Obtained vectors of particle velocity in each point provided a basis for visualizations of the sound intensity. Visual presentations consist of such forms as the sound intensity streamlines, intensity vectors and intensity magnitude distribution. Graphs and charts were calculated using the Paraview software (AHREns et al., 2005). Visualizations of sound intensity field both for open pipe and stopped pipe are presented in Figs. 10 to 16 . Intensity magnitude was calculated using formula (6), presented in Sec. 2. Figures 10 and 11 present cross sections taken in the middle the organ pipe $(x=1000)$. The streamlines and intensity vectors were in those two cases calculated for two components, $I_{y}$ and $I_{z}$.

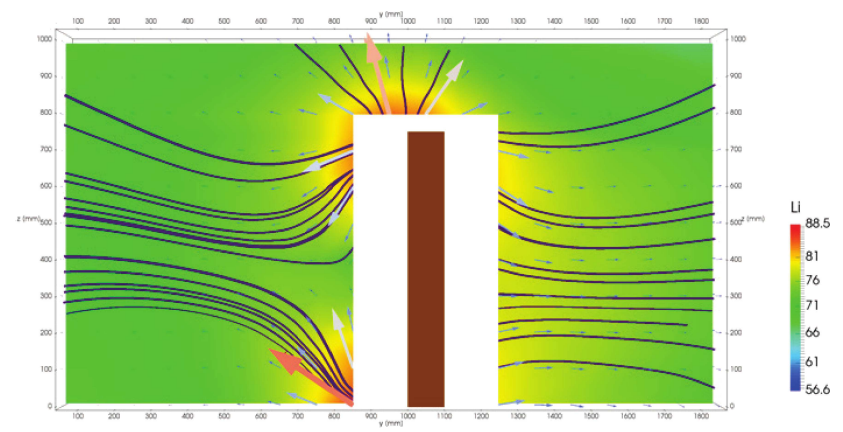

Fig. 10. Sound intensity distribution around open organ pipe, the view from right side of the pipe. The intensity vector arrows and streamlines are also depicted.

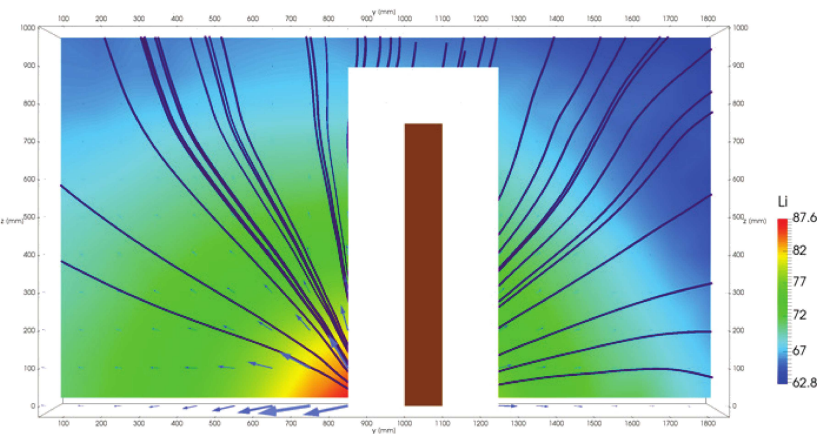

Fig. 11. Sound intensity distribution around stopped organ pipe, the view from right side of the pipe. The intensity vector arrows and streamlines are also depicted.

In the case of the stopped pipes, the acoustic energy is concentrated in the area of the lip, where 
the emission of an acoustic wave happens. This is clearly shown in the diagrams depicting the direction of propagation of the acoustic energy, illustrated as streamlines (Figs. 10 and 11). Lines seem to flow only from the lower part of the instrument. We observe the highest values of sound intensity near the gap through which the air exits. The stream of the acoustic wave starting from the lowest measurement points ( $\mathrm{Z}$ axis $=0$ ) is directed to the front of the instrument. With the increase of the height (increase of the $\mathrm{Z}$-axis values) of the measuring point, we observe the increase in the vertical direction sound propagation.

On the next chart, showing the view from the top, one can see the concentration of acoustic energy in the front of the instrument, where the exit slot (mouth) is located (Fig. 12). A completely different distribution of directions of propagation of acoustic energy was observed for the open pipes. In this case, the two main areas of concentration of the acoustic energy are clearly visible (Fig. 13). They are located at the lip (bottom of the pipes) and at the air exit at the top of the instrument. The sound energy emission occurs mainly in those areas. Then, the sound energy dispersed from these areas may enter into the mutual interaction, re- sulting in a varied arrangement of the propagation of acoustic energy, depending on the height of the measuring point (Figs. 14 and 15).

Such a distribution of directions is observed as high as the total height of the considered pipe. In the area above the pipe, the streams of acoustic energy are definitely directed upwards. Another difference is seen in the top view. For open pipes, even distribution of acoustic energy around the instrument is observed. The acoustic energy is focused not only in front of the instrument (as was the case with the stopped pipe) but is spread quite evenly both in front of the instrument, as well as to the left and right side. The lowest values of sound intensity were observed in the rear of the instrument.

The measurements of the sound intensity distribution for the considered variations of the organ pipe revealed a different distribution of the direction of propagation of acoustic energy. The fact of opening or stopping the pipe affects radically not only the frequency of the generated sound, but also the sound pressure level and direction of propagation of acoustic energy. In order to observe various propagation directions, the streamline of the two types of pipes is combined in one diagram (Fig. 16).
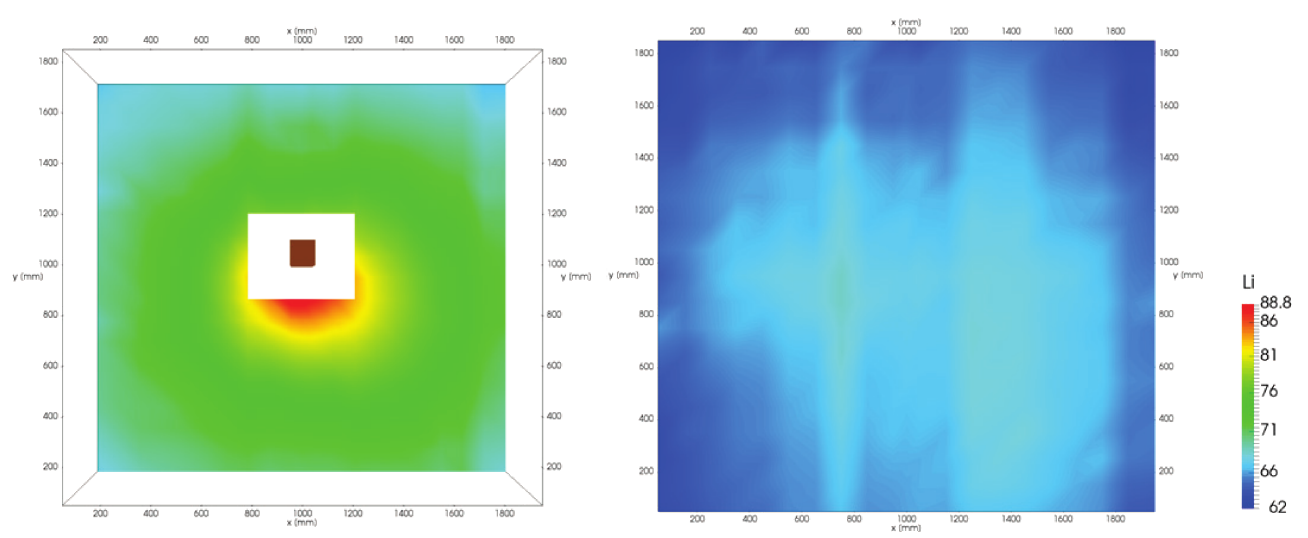

Fig. 12. Sound intensity distribution around stopped organ pipe: a) the magnitude of the sound intensity measured at $z=0, \mathrm{~b})$ the intensity distribution above the organ pipe $(z=800)$.
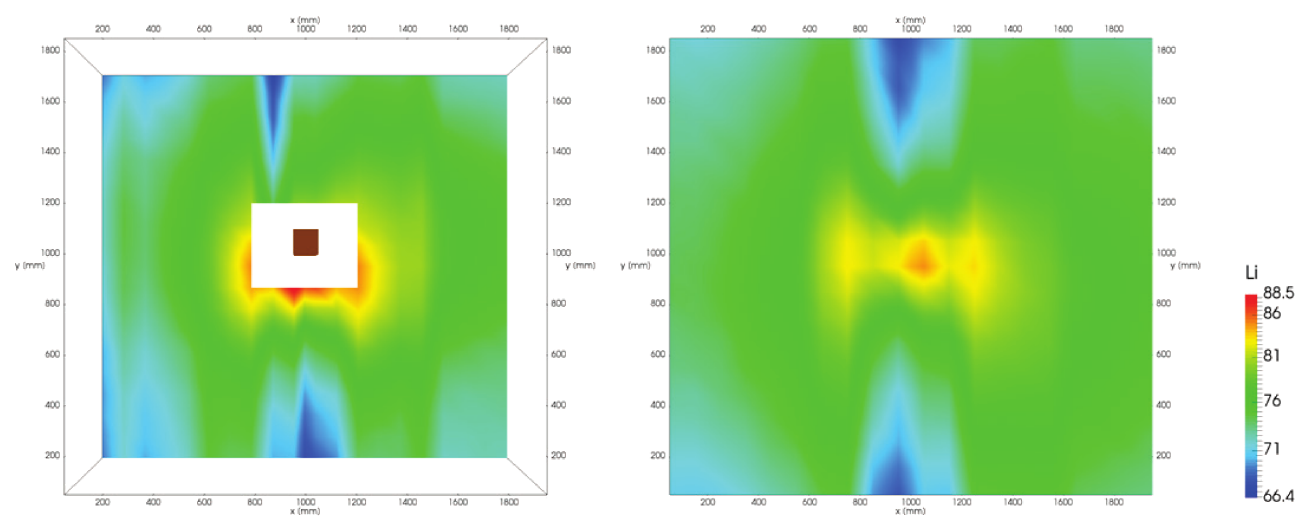

Fig. 13. Sound intensity distribution around open organ pipe: a) the magnitude of the sound intensity measured at $z=0, \mathrm{~b})$ the intensity distribution above the organ pipe $(z=800)$. 


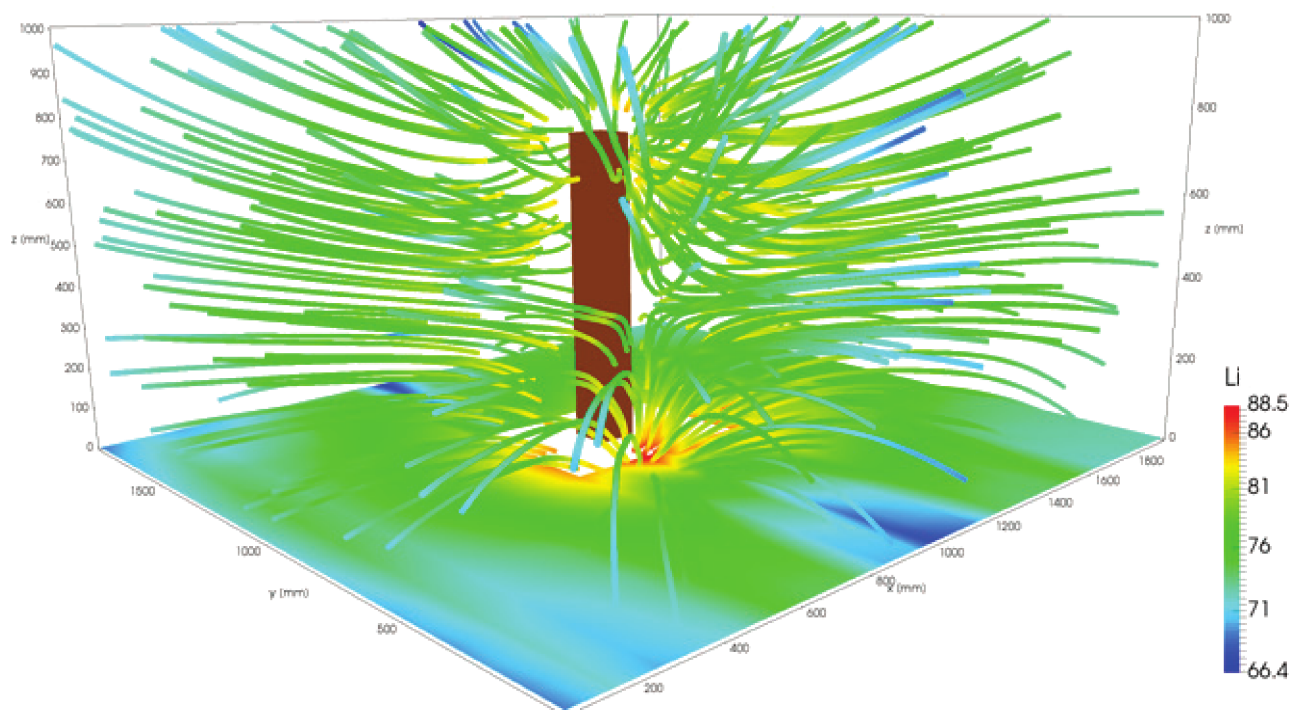

Fig. 14. Sound intensity distribution around open organ pipe. The sound intensity level was shown at the bottom. Streamlines which indicate the direction of the acoustic energy flow were also presented.

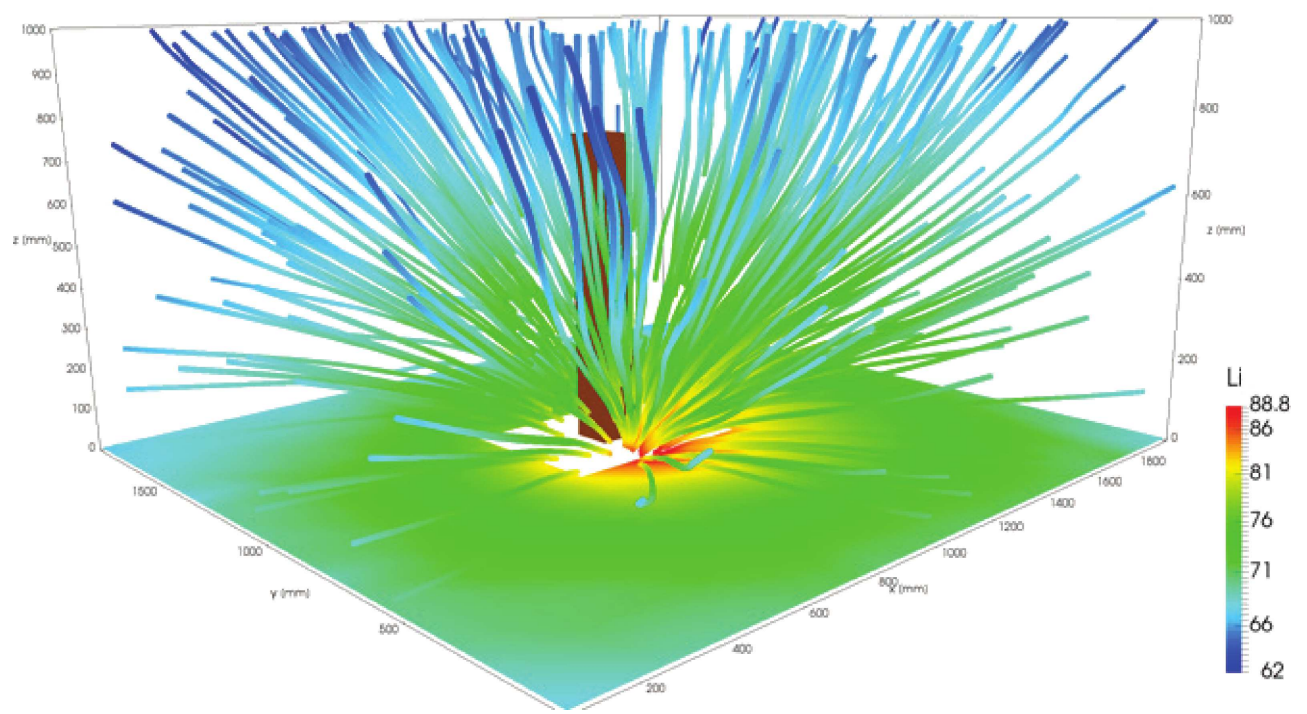

Fig. 15. Sound intensity distribution around stopped organ pipe. The sound intensity level was shown at the bottom. Streamlines which indicate the direction of the acoustic energy flow were also presented.

a)

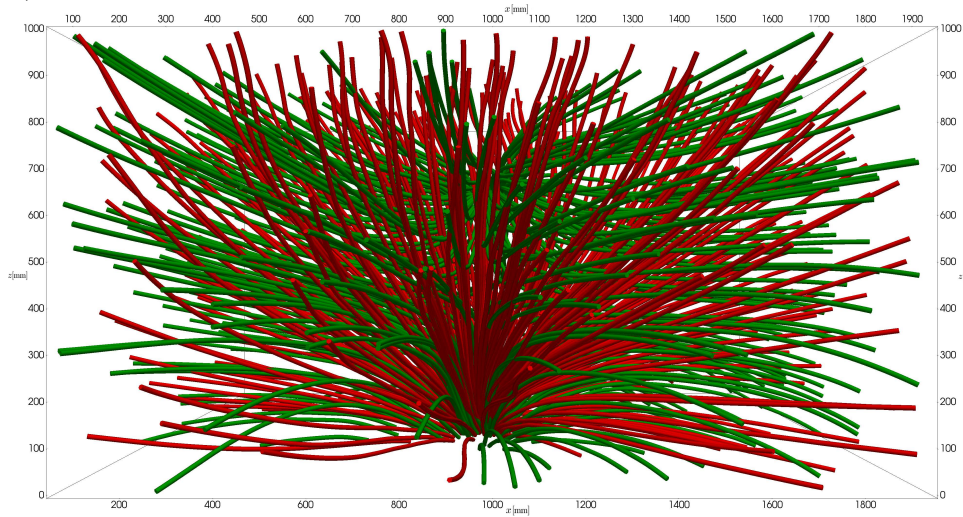

b)

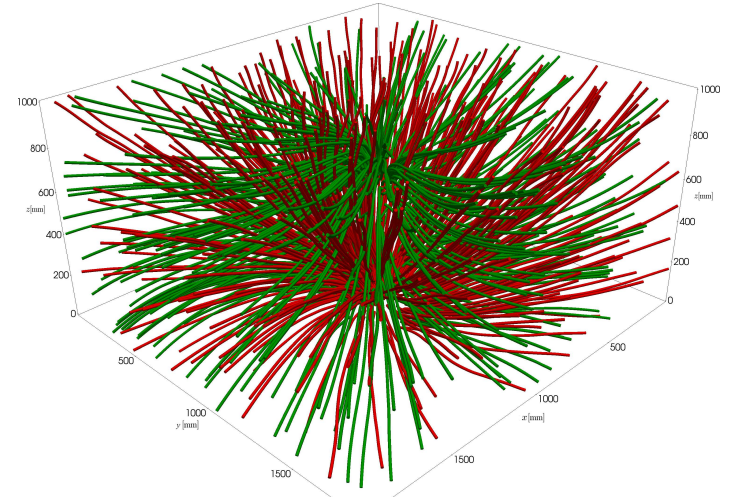

Fig. 16. Combined presentation of the streamlines calculated for open organ pipe (green lines) and stopped organ pipe (red lines): a) the view in front of the pipes, b) perspective view, camera was placed to the right side above the organ pipes. 
The differences in radiation directivity disclosed through the measurements of both pipe variation are also of importance for the reception of sound produced by instruments composed of multiple pipes. It is expected that in the case of organs whose individual pipes are open, the direct sound is propagated to the right and slightly to the bottom of the instrument. Thanks to that, the listener (usually positioned below the mouth of pipes) is first reached by the direct wave and after some time by early reflections and finally reverberation. As a result, the musical message should be more readable than when the stopped pipes are used.

\section{Conclusions}

The application of sound intensity measurement technique for acoustic energy distribution around organ pipes was presented in the paper. During measurements the same wooden pipe (Bourdon, with and without the tuning stopper) was used. It was originally stopped, its full length is $88 \mathrm{~cm}(99 \mathrm{~cm}$ with a tuning stopper). Such configurations give us possibility to obtain results that can more clearly show the differences in sound intensity distributions in case of two organ pipe types. Authors precisely prepare the grid of measurement points and performed the sound intensity measurements using Acoustic Vector Sensors and Cartesian Robot. All measurements were performed in anechoic chamber (free field conditions).

On the basis of performed recording, the sound intensity distributions around organ pipes were obtained and visualized. Directions of the acoustic energy flow were also presented. The sound intensity technique was sufficiently accurate to be applied for determination of acoustic energy distribution and sound transmission around organ pipes. On the basis of obtained results, several differences in examined organ pipes were observed. First of all, for stopped organ pipe, the total sound pressure level measured in front of the lips was greater $(99.9 \mathrm{~dB})$ than for open version $(97.8 \mathrm{~dB})$. For open pipe, another sound source was present at the top of the pipe. This is the most important difference between the pipes. This difference in construction of the pipes evoked essential differences in the acoustic energy distribution around them. For the stopped pipe, we observed one main acoustic energy source, for the open one, there are two sound sources. The spatial arrangement of the sound sources affects the sound intensity distribution. For that reason, for the open version the streamlines in front of the pipe are propagated horizontally and in a greater distance from the pipe are directed downwards. For the stopped version, we observed completely different sound intensity distribution. In this configuration the streamlines of the acoustic flow were directed upwards. The applied sound intensity measurement technique gives us possibility to obtain and present the detailed information about radiation directivity of the considered musical instruments. Such technique can be also applied for other kinds of musical instruments.

\section{Acknowledgments}

The project was partially funded by the National Science Centre on the basis of the decision number DEC-2012/05/B/ST7/02151.

\section{References}

1. Ahrens J., Geveci B., Law C. (2005), ParaView: An End-User Tool for Large-Data Visualization, [in:] Visualization Handbook, C. Hansen, C. Johnson [Eds.], Butterworth-Heinemann, Burlington, 2005, pp. 717731, LXX-LXXII.

2. DE BREE H.-E. (2003), The Microflown: an acoustic particle velocity sensor, Acoust. Aust., 31, 3, 91-94.

3. Coltman J.W. (1968), Sounding mechanism of the flute and organ pipe, Journal of the Acoustical Society of America, 44, 983-992.

4. Colmman J.W. (1969), Sound radiation from the mouth of an organ pipe, Journal of the Acoustical Society of America, 46, 477.

5. FAну F.J. (1995), Sound intensity, E \& F.N. Spon.

6. Fletcher N., Rossing T.D. (1998), The Physics of Musical Instruments, Springer Science+Business Media, New York.

7. Fletcher N.H. (1976), Sound production by organ flue pipes, Journal of the Acoustical Society of America, 60, 926-936.

8. Fletcher N.H., Thwaites S. (1983), The physics of organ pipes, Scientific American, 248, 94-103.

9. Gauthier P.-A., Camier C., Padois T., Pasco Y., Berry A. (2015), Sound Field Reproduction of Real Flight Recordings in Aircraft Cabin Mock-Up, J. Audio Eng. Soc., 63, 1/2, 2015 January/February, 1-20, https://doi.org/10.17743/jaes.2015.0001.

10. Jacobsen F. (2011), Sound Intensity and its Measurement and Applications, Acoustic Technology, Department of Electrical Engineering Technical University of Denmark.

11. Jacobsen F., De Bree H.-E. (2005), A comparison of two different sound intensity measurement principles, Journal of the Acoustical Society of America, 118, 1510-1517.

12. Kotus J. (2015), Multiple sound sources localization in free field using acoustic vector sensor, Multimedia Tools and Applications, 74, 12, 4235-4251.

13. Kotus J., Kostek B. (2015), Measurements and visualization of sound intensity around the human head in free field using acoustic vector sensor, J. Audio Eng. Soc., 63, 1/2, 99-109.

14. Kotus J., Odya P., Kostek B. (2015a), Measurements and visualization of sound field distribution 
around organ pipe, Proceedings of the 19th IEEE Conference SPA 2015, Signal Processing: Algorithms, Architectures, Arrangements, and Applications, pp. 145150, Poznań.

15. Kotus J., Odya P., Szczodrak M., Kostek B. (2015b), 3D Sound Intensity Measurement Around Organ Pipes Using Acoustic Vector Sensors, [in:] Progress of Acoustics, Opielinski K.J. [Ed.], pp. 105-117, Polish Acoustical Society, Wroclaw Davison, Wroclaw.

16. Kotus J., Czyżewski A., Kostek B. (2016), 3D Acoustic Field Intensity Probe Design and Measurements, Archives of Acoustics, 41, 4, 701-711, DOI: 10.1515/aoa-2016-0067.

17. Kuang W., Angster J., Yang J., Miklos A. (2015), Sound Radiation Pattern of the Sheng Pipes, DAGA 2015, Nürnberg, Germany.

18. MickieWICZ W. (2014), Visualization of sound generation mechanism in organ flue pipe by means of particle image velocimetry, 7th Forum Acusticum 2014, Krakow, Poland.

19. Mickiewicz W. (2015), Particle Image Velocimetry and Proper Orthogonal Decomposition Applied to Aerodynamic Sound Source Region Visualization in Organ Flue Pipe, Archives of Acoustics, 40, 4, 475-484, DOI: 10.1515/aoa-2015-0047.

20. Nagata S., Furihata K., Wada T., Asano D.K., YANAGisawa T. (2005), A three-dimensional sound intensity measurement system for sound source identification and sound power determination by $\mathrm{ln}$ models, J. Acoust. Soc. Am., 118, 6, 3691-3705.

21. Polychronopoulos S., Skarlatos D., MourJopoulos J. (2014), Efficient Filter-Based Model for Resonator Panel Absorbers, J. Audio Eng. Soc., 62, 1/2, 14-24, https://doi.org/10.17743/jaes.2014.0005.

22. Rucz P., Trommer T., Angster J., Miklós A., Augusztinovicz F. (2013), Sound design of chimney pipes by optimization of their resonators, J. Acoust. Soc. Am., 133.1, C: 1, 529-537.

23. Rucz P., Augusztinovicz F., Angster J., PreuksCHAT T., Miklós A. (2014), Acoustic behavior of tuning slots of labial organ pipes, J. Acoust. Soc. Am., 135.5, 3056-3065.

24. Rucz P., Augusztinovicz F., Angster J., PreuksCHAT T., Miklós A. (2015), A finite element model of the tuning slot of labial organ pipes, J. Acoust. Soc. Am., 137.3, 1226-1237.

25. Rucz P. (2015), Innovative methods for the sound design of organ pipes, Ph.D. Booklet, Budapest University of Technology and Economics Faculty of Electrical Engineering And Informatics Doctoral School of Electrical Engineering, Budapest.

26. Szczodrak M., Kurowski A., Kotus J., CzyżewsKI A., KosteK B. (2016), A system for acoustic field measurement employing cartesian robot, Metrology and Measurement Systems, 23, 3, 333-343.

27. Steenbrugge D. (2011), Fluid mechanical aspects of open- and closed-toe flue organ pipe voicing, Sustainable Construction and Design, 2, 284-295.

28. VAN DE Perre G. (2011), Experimental study of organ pipe behavior using optical measurement techniques, M.Eng. Thesis, Department Mechanical of Engineering, Vrije Universiteit Brussel.

29. Weyna S. (2003), Identification of Reflection and Scattering Effects in Real Acoustic Flow Field, Archives of Acoustics, 28, 3, 191-203.

30. Weyna S. (2010), An Acoustics Intensity Based Investigation of the Energy Flow Over the Barriers, Acta Physica Polonica A. 1, Acoustic and Biomedical Engineering, 118, 172-178.

31. Weyna S., Mickiewicz W. (2014), Multi-modal acoustic flow decomposition examined in a hard walled cylindrical duct, Archives of Acoustics, 39, 2, 289-296, DOI: 10.2478/aoa-2014-0033. 